\title{
Scepticisme, fidéisme et évidentialisme : oppositions et origines
}

\author{
ARTŪRS LOGINS Université de Genève
}

RÉSUMÉ : Les principales attitudes adoptées devant les questions centrales de l'épistémologie générale (en particulier la question de la croyance justifiée) sont selon nous l'évidentialisme, le scepticisme (pyrrhonien) et le fidéisme. Dans cet article, nous présentons d'une part les arguments permettant d'accepter une forme de l'évidentialisme selon laquelle aucune croyance fausse ne peut être justifiée sur la base de raisons probantes. D'autre part, nous proposons de considérer l'aspect historique de l'émergence de l'évidentialisme au début des Lumières. Nous explorons un cas particulier qui témoigne de l'émergence de l'évidentialisme moderne comme opposition au fidéisme. Il s'agit de l'opposition entre Pierre Bayle et Jean-Pierre de Crousaz.

ABSTRACT: I maintain that among the main views concerning the central questions of epistemology (in particular, the question of justified belief) are evidentialism, (Pyrrhonian) scepticism and fideism. In this paper, I first present the arguments in favour of a form of evidentialism, according to which no false belief can be epistemically justified on the basis of evidence. Second, I consider the historical emergence of evidentialism during the period of the early Enlightenment. In particular, I explore the disagreement between Pierre Bayle and Jean-Pierre de Crousaz, which demonstrates the emergence of the modern opposition between evidentialism and fideism.

\section{Introduction}

Qu'est-ce qu'une croyance raisonnable ${ }^{1,2}$ ? Nos croyances sont-elles pour la plupart raisonnables?

Dialogue 51 (2012), 613-642.

(C) Canadian Philosophical Association/Association canadienne de philosophie 2012 doi:10.1017/S0012217313000292 
Du point de vue de nos intuitions ordinaires, ces questions peuvent paraître pour le moins suspicieuses. «Bien sûr que la plupart de nos croyances sont raisonnables! Une croyance raisonnable? Ce sont celles que nous avons la plupart du temps», pourrait-t-on rétorquer depuis la perspective de l'homme de la rue. Mais en vertu de quel principe cette réponse tient-elle?

À ces questions s'ajoute un autre type d'interrogation concernant la connaissance. Est-il vrai que nous possédons une quelconque connaissance dans ce monde tellement trompeur? Par ailleurs, n'est-t-il pas exclu que nous nous soyons trompés de manière radicale, de telle façon que la majeure partie de ce que nous croyons n'est qu'illusion?

De telles questions occupaient une grande partie de la discussion philosophique durant l'époque moderne. Depuis, elles n'ont jamais quitté l'avant de la scène philosophique. C'est pourquoi ces mêmes questionnements sont présents dans les débats de l'épistémologie contemporaine. L'épistémologie est donc la discipline où l'on s'interroge sur la possibilité même d'avoir une connaissance ${ }^{3}$ et sur la question de la croyance justifiée ${ }^{4}$.

Néanmoins, il serait injuste d'affirmer que les questions concernant le statut de nos croyances ou encore les défis sceptiques posés aux connaissances, même celles concernant le monde extérieur, apparaissent seulement à l'époque moderne 5 .

La principale question qui nous intéresse est celle de la croyance raisonnable. Les deux problématiques susmentionnées sont liées même si elles sont fondamentalement indépendantes. Elles sont liées car si l'on suppose que nos croyances ne peuvent pas être raisonnables, alors nous sommes plus aptes à embrasser le point de vue sceptique concernant la connaissance.

Les réponses traditionnelles aux problématiques centrales de l'épistémologie peuvent être classées en trois groupes : - les positions qui acceptent le scepticisme dans l'une de ses formes; - les positions dogmatistes qui proposent des réponses claires mais (probablement) arbitraires; - les positions qui tentent de combiner les deux options précédentes, ou, du moins, qui tentent de rendre justice et d'expliquer les intuitions qui soutiennent les deux options précédentes.

L'évidentialisme épistémologique moderne — la théorie selon laquelle la justification de nos croyances repose sur la totalité des raisons probantes ${ }^{6}$ que nous possédons - est une réponse du troisième type. Elle semble par ailleurs être très populaire dans l'épistémologie contemporaine.

Il s'avère difficile de caractériser ce qu'est exactement la doctrine évidentialiste. Car l'évidentialisme peut avoir plusieurs formes.

Une des versions qui semble être parmi les plus populaires en épistémologie contemporaine est ce que nous appellerons «l'évidentialisme tolérant».

L'évidentialisme tolérant : la croyance que $p$ de la personne $S$ est justifiée au moment $t$ si et seulement si la totalité des raisons probantes («evidence») que possède $S$ au moment $t$ soutient $p$ ET il est possible que $p$ soit faux ${ }^{7}$.

Cette version tolérante de l'évidentialisme semble motivée par l'observation régulière de nos capacités d'être trompés à notre insu ou de tomber dans l'erreur 
non volontairement. On peut considérer, assez intuitivement, la situation où nous demandons l'heure à quelqu'un dans la rue, et tombons par malchance sur une personne malveillante ou simplement ignorante de la vérité, qui nous donne une mauvaise réponse en nous mettant ainsi dans l'erreur. Nous nous jugeons néanmoins raisonnables ou justifiés.

À cette version, nous opposons une autre position évidentialiste: «l'évidentialisme de tolérance zéro».

L'évidentialisme de tolérance zéro : la croyance que $p$ de la personne $S$ est justifiée au moment $t$ si et seulement si la totalité des raisons probantes que possède $S$ au moment $t$ soutient $p$ ET $p$ n'est pas faux. (Il s'agit alors d'une position de «tolérance zéro», car les raisons qui ne nous «conduisent» pas vers la vérité ne seront pas tolérées sur le plan de l'évaluation doxastique).

La motivation principale pour préférer l'évidentialisme de tolérance zéro vient de notre usage linguistique ordinaire (ou de sens commun) et des domaines scientifiques dans lesquels il n'est pas cohérent de parler de «fausses raisons probantes» («misleading evidence» ou «false evidence»). En science, par exemple, il n'est pas commun, du moins si l'on n'est pas influencé par certaines idées relativistes de certains philosophes des sciences de la fin du $\mathrm{XX}^{\mathrm{e}}$ siècle, de dire : «La théorie $\mathrm{T}$ reçoit une confirmation à un degré élevé par les données (raisons probantes), même si T est fausse», ou encore : «Il est justifié de croire que la théorie de l'éther est confirmée par les données, même si elle est fausse». Dans le langage ordinaire, en accord avec le sens commun, il semble étrange d'affirmer sans ironie quelque chose comme : «Tu te trompes de chemin, je le sais. C'est mon quartier, il faut prendre la route de gauche. Mais tu as des raisons probantes d'avoir choisi cette route, même si elles sont fausses», ou encore : «Je sais que la Terre tourne autour du Soleil. Mais je suis justifié de croire que le Soleil tourne autour de la Terre, car j'ai pour cela des raisons probantes trompeuses». Face à de tels exemples, il semble que des raisons probantes fausses ou trompeuses ne peuvent pas exister. En tout cas, on ne dira jamais que des raisons probantes fausses confirment notre hypothèse ou justifient notre croyance. Au contraire, si l'on s'appuie sur des raisons probantes, c'est parce que nous croyons qu'elles confirment ou soutiennent la vérité de ce que nous croyons. D'habitude, on fait appel aux raisons probantes ou l'on demande des raisons probantes parce que nous voulons établir la vérité; nous n'admettons pas ordinairement qu'une proposition fausse puisse être confirmée par des raisons probantes.

Toutefois, le défi principal de l'évidentialisme de tolérance zéro reste d'expliquer pourquoi certaines de nos intuitions semblent soutenir plutôt une version tolérante de l'évidentialisme. Notre réponse à ce défi consiste à indiquer que l'intuition qui nous semble soutenir l'évidentialisme tolérant soutient en réalité une autre thèse, à savoir le principe de la prudence doxastique — voulant que nous ne sommes pas nécessairement en mesure de trouver l'évaluation doxastique de nos croyances qui correspond exactement à la réalité. La tentation de passer de la prudence doxastique à la tolérance évidentialiste est importante, 
pourtant c'est un pas non nécessaire et comme on le verra par la suite, un pas qui nous désarme face au défi sceptique.

La thèse que nous défendons dans le présent article est donc que seul l'évidentialisme de tolérance zéro est en mesure d'opposer au sceptique une réponse qui tienne.

Notre deuxième thèse est historique et concerne l'apparition de l'évidentialisme tolérant. Elle peut s'énoncer comme suit : l'évidentialisme moderne a émergé plus comme une réaction au fidéisme (ou plus précisément au fidéisme sceptique - i.e. fidéisme intégrant les intuitions sceptiques) que comme une réponse au scepticisme. Différents arguments sceptiques circulaient au Moyen Âge même si les œuvres de Sextus Empiricus n'étaient pas très accessibles. Mais ces questions ne produisaient pas ce qu'on appelle l'évidentialisme au sens moderne. En revanche, après la redécouverte de Sextus, la naissance du scepticisme moderne chrétien, soit de problématiques sceptiques encadrées dans le domaine théologique, a été décisive dans la montée du fidéisme. Étant l'opposé de l'évidentialisme, le fidéisme a joué un rôle primordial dans l'élaboration de celui-ci. Les disciples de Descartes comme Jean-Pierre de Crousaz ont contribué à l'émergence et à la popularisation de l'évidentialisme en combattant le fidéisme sceptique et en s'appuyant sur l'idée voulant que l'activité de la raison pouvait donner accès aux raisons probantes. Et c'est en complétant cette attitude évidentialiste générale issue de l'opposition au fidéisme et enracinée dans la confiance en la raison avec le principe de tolérance doxastique - selon lequel le statut (justifié ou non-justifié) d'une croyance n'est pas nécessairement dépendant de la valeur de vérité du contenu de cette croyance — que l'évidentialisme a pris le tournant tolérant.

Une perspective permettant de remplacer l'évidentialisme tolérant moderne, plus proche de l'évidentialisme de tolérance zéro, peut être discernée dans les écrits de philosophes dits du «sens commun» comme Thomas Reid ou Claude Buffier (voir p. ex. Rysiew, 2005). Même si nous nous sentons assez proches de cette version de l'évidentialisme, nous n'abordons pas directement cette position ici.

Notre objectif global, qui concerne l'épistémologie générale, consiste à défendre une forme générale de l'évidentialisme de tolérance zéro, et ce, de façon historiquement informée, c'est-à-dire en renforçant notre position par un argument provenant de l'histoire de la philosophie.

Cet article se développera donc en deux temps. 1) Nous considérerons d'abord le scepticisme, le fidéisme et l'évidentialisme relativement aux questions fondamentales de l'épistémologie (moderne). Quels sont les arguments et les options permettant de défendre le scepticisme et comment le fidéisme a-t-il pu s'installer en filigrane du scepticisme? Nous verrons ensuite pourquoi le fidéisme n'est pas une position plus acceptable que le scepticisme si nous ne sommes pas convaincus par les arguments sceptiques. Nous proposerons enfin les raisons qui nous poussent à préférer l'évidentialisme de tolérance zéro, et à rejeter l'évidentialisme tolérant (section 2). 2) Notre deuxième objectif est d'affirmer 
que l'évidentialisme a pris son essor grâce aux positions fidéistes. Pour montrer cela, nous présentons un exemple de discussion ayant favorisé cette émergence : une discussion entre Pierre Bayle et Jean-Pierre de Crousaz - un cas concret de l'opposition entre l'évidentialisme et le fidéisme. Nous espérons que cette seconde considération permettra de montrer encore mieux pourquoi nous devrions choisir l'évidentialisme de tolérance zéro, en révélant que le détour par l'évidentialisme tolérant n'était pas nécessaire. Pour contrer le scepticisme et le fidéisme, seul le principe de prudence doxastique aurait suffi, sans qu'il fût nécessaire de concéder au sceptique ou au fidéiste tolérant le pas vers la tolérance évidentialiste.

\section{Le scepticisme, le fidéisme et l'évidentialisme}

Dans les temps anciens déjà, le scepticisme se déclinait en deux versions. Pour cerner ces deux options, nous devons tout d'abord comprendre quelles sont les attitudes possibles envers les propositions ${ }^{8}$. Face à une proposition quelconque (ou à une classe de propositions) on peut avoir trois attitudes :

1) Il nous est possible d'accepter (de consentir) que nous pouvons connaître cette proposition (ce que la proposition exprime). Cette position a été appelée dans l'Antiquité la position dogmatique ou non-sceptique;

2) Nous pouvons aussi accepter que nous ne pouvons pas avoir de connaissance de ces propositions - c'est la position du scepticisme académique;

3) Nous pouvons n'accepter aucune des deux premières possibilités et suspendre notre jugement vis-à-vis de la possibilité de connaissance de ces propositions — c'est la position du scepticisme pyrrhonien ${ }^{9}$.

Notre centre d'intérêt dans cet article concernera surtout la troisième option, en grande partie parce qu'il nous semble que c'est le défi pyrrhonien qui, s'il n'est pas bloqué, cause les dégâts les plus vastes à toute entreprise philosophique qui s'appuie sur la présupposition de la possibilité de la connaissance. Mais la position pyrrhonienne nous intéresse surtout dans la mesure où elle, ou plus précisément une de ses variations, a permis l'émergence de ce que je qualifierai d'évidentialisme tolérant moderne appliqué à la justification des croyances.

C'est à la fin de la Renaissance et au début de l'époque moderne que des positions de type pyrrhonien sont devenues largement populaires dans le débat entourant deux questions épistémologiques : la question de la justification des croyances et la question de la possibilité de la connaissance. Cette émergence de variations pyrrhoniennes est largement due à la publication des œuvres de Sextus Empiricus par Henri Estienne en 1562. Mais c'est surtout la lecture de celles-ci et les thèses philosophiques propres de Michel de Montaigne, Pierre Charron, Jean-Pierre Camus, Francisco Sanchez et François de La Mothe Le Vayer qui ont donné au paysage des débats philosophiques concernant la connaissance des «couleurs» sceptiques (voir notamment Popkin, 1963). 
Avant de présenter en détail les positions sceptiques de l'époque moderne et leurs particularités, il serait utile d'aborder quelques aspects essentiels du scepticisme pyrrhonien en général. Comment un sceptique pyrrhonien répond-il aux problématiques des croyances justifiées et de la possibilité de connaissance?

On peut caractériser ce qui est essentiel au scepticisme pyrrhonien de la façon suivante.

\section{Scepticisme pyrrhonien}

Pour toute proposition quelconque $p$, si $p$ porte sur quelque chose dont nous pouvons juger, alors nous devons suspendre notre jugement concernant $p$ et concernant non- $p$ (nous ne devons affirmer ni $p$, ni non- $p$ ), puisque :

i)

pour tout $p$ à propos duquel nous pouvons juger, il existe des arguments soutenant $p$ et des arguments soutenant non- $p$.

Le scepticisme pyrrhonien ainsi présenté permet de répondre aux questions épistémologiques que nous avons posées. Du point de vue sceptique, l'approche pyrrhonienne semble même avoir un avantage sur le scepticisme académique qui était par ailleurs déjà largement discuté au Moyen Âge depuis saint Augustin. Là où le scepticisme académique répond par la négative à la question concernant la possibilité de la connaissance, une réponse qui pourrait être qualifiée d'incohérente, voire de contradictoire ${ }^{10}$, le scepticisme pyrrhonien n'affirme pas qu'il est impossible de connaître. Les pyrrhoniens antiques ainsi que modernes, comme Montaigne, voient en effet dans le scepticisme académique une sorte de dogmatisme déguisé. Le scepticisme pyrrhonien aspire à une réponse plus radicale concernant la question de la possibilité de la connaissance. Un pyrrhonien concédera que dans l'absolu, il est peut-être possible de connaître, mais précisera que nous ne pouvons en aucune manière accepter ou nier que cela soit le cas.

Le scepticisme pyrrhonien, du moins dans sa forme moderne, est le plus radical dans la mesure où il nie la possibilité même des croyances justifiées. Il implique par conséquent que nous devons toujours suspendre notre jugement. Or, il n'est pas possible de donner des raisons ultimes pour justifier nos croyances. Cette conclusion que tire le pyrrhonien découle de l'argument connu sous le nom de trilemme d'Agrippa ${ }^{11}$. Afin d'avoir une croyance justifiée, nous sommes obligés de choisir parmi trois possibilités incompatibles ${ }^{12}$ : soit nos croyances sont justifiées grâce à une chaîne de raisons justifiantes qui remonte à l'infini, soit nos croyances sont justifiées grâce à une chaîne de raisons circulaire, soit nos croyances sont justifiées grâce à une chaîne de raisons qui s'arrête de façon arbitraire à un certain niveau. Or, les trois options semblent présenter des difficultés. Pour un pyrrhonien, la meilleure solution à ce trilemme est d'admettre 
que nos croyances ne sont pas justifiées et que nous ferions mieux de suspendre nos jugements. Nous verrons cet argument plus en détail, mais avant cela, considérons d'abord quelles sont les intuitions qui motivent l'attitude sceptique pyrrhonienne.

Nous avons vu que les sceptiques pyrrhoniens anciens comme modernes peuvent répondre par la négative à la question de la justification, mais par l'affirmative à la question de la connaissance dans l'absolu. Les motivations théoriques semblent pour autant ne pas être les mêmes chez les uns et chez les autres. La motivation fondamentale derrière la position des sceptiques anciens est l'intuition concernant le critère de vérité de la croyance. Les sceptiques anciens affirment, en opposition aux stoïciens et aux épicuriens, qu'il n'existe pas de signe permettant d'identifier une impression comme vraie et de former des croyances sur les bases de ces standards (Vogt, 2011). Chez les sceptiques modernes, en revanche, l'intuition principale sera la possibilité d'être trompé par Dieu ou par un malin génie de sorte que tout ce que nous croyons est en dernière instance faux ${ }^{13}$.

Dans ce qui suit, nous abordons une interprétation du trilemme d'Agrippa plutôt proche de l'esprit du scepticisme moderne et plus proche des discussions contemporaines. Toutefois, il est possible de changer la terminologie de sorte que l'argument soit plus proche des idées des sceptiques anciens, notamment en remplaçant la question de la croyance justifiée par la question de la croyance qui respecte le critère de vérité ${ }^{14}$.

\section{Trilemme d'Agrippa dans l'argumentation du scepticisme pyrrhonien}

Il existe seulement trois attitudes possibles (mutuellement exclusives) vis-à-vis de la justification de nos croyances. 1) Soit nous nous arrêtons à un moment précis de manière arbitraire dans la recherche de croyances justifiées en considérant qu'il y a des croyances qui sont justifiées et qui justifient d'autres croyances sans qu'elles-mêmes aient besoin d'avoir à leur fondement d'autres croyances qui les justifient. Mais ce moment est arbitraire, car il est toujours possible de demander des raisons encore plus fondamentales pour qu'une croyance soit justifiée. Cette réponse est qualifiée de dogmatique par les sceptiques pyrrhoniens. 2) Soit nous acceptons que toutes les croyances ont besoin d'autres croyances justifiées comme ce qui les justifie. Cela implique une sorte de circularité, ce qui n'est pas souhaitable, car admettre la circularité semble impliquer une erreur de raisonnement. Pour le dire simplement, la difficulté est ici d'expliquer, sans commettre un raisonnement fallacieux, comment une croyance qui est censée en justifier une autre pourrait elle-même être justifiée par la croyance qu'elle est en train de justifier. 3) Soit nous acceptons la régression à l'infini dans la justification des croyances. Dans ce cas, nous ne pourrons jamais nous arrêter pour affirmer qu'une croyance est, effectivement, ultimement justifiée, car nous devrons toujours chercher d'autres croyances pour justifier une croyance, et cela à l'infini. Les sceptiques pyrrhoniens pensent que cette option doit être choisie en ce qui concerne la possibilité de croyances justifiées. Cela implique selon eux que nous ne pouvons jamais avoir de croyances ultimement justifiées, et même que nous 
ne devons jamais acquérir des croyances. Sextus Empiricus affirme que devant ces problèmes, nous devons suspendre notre jugement, par exemple vis-à-vis d'une proposition comme «le miel est doux».

Il existe une objection à cette position aussi ancienne que le scepticisme lui-même. Il s'agit de l'objection à partir de l'apraxia, autrement dit à partir de l'impossibilité de l'action sans la base des croyances, aussi connue comme l'objection de l'impossibilité de vivre en sceptique. Si nous devons suspendre notre jugement à propos de toute proposition, alors nous ne pourrons réaliser aucune action. Or toute action, qu'il s'agisse de manger, de discuter avec autrui, etc., implique des croyances sur la base desquelles nous agissons. S'il faut suspendre le jugement et ne pas avoir de croyances du tout, alors il semble que nous sommes condamnés à mourir de faim et de soif, à tomber du haut des falaises ou à être écrasés par des moyens de transport.

La réponse sceptique à cette accusation consiste à dire qu'il est faux de dire qu'une croyance est nécessaire pour toute action. Il est possible que les actions soient guidées par des apparences, ou par ce qui semble plausible (Vogt, 2011). Les croyances pleines, comme on les avait définies, ne sont pas (toujours) nécessaires pour guider une action.

Un autre type d'objection contre la position sceptique pyrrhonienne nous semble plus radical encore. Si nous acceptons que la caractéristique commune des différents états doxastiques (la croyance, la non-croyance ou la suspension de jugement) est le fait d'accepter une proposition $p$, non- $p$, ou ni $p$ ni non- $p$ et de la tenir pour vraie, nous sommes en mesure de montrer les implications paradoxales du pyrrhonisme. Dans ce qui suit, nous proposons deux objections au scepticisme pyrrhonien.

La première objection est la suivante. Si nous acceptons que, d'une part, la suspension de jugement est un état doxastique de même nature que la croyance, et, d'autre part, que face au trilemme d'Agrippa nous devons suspendre nos jugements (ne devons pas croire que $p$ ), alors nous aboutissons à une situation paradoxale, semblable à celle du paradoxe de Moore. Nous aboutissons à la situation dans laquelle nous nions la qualité pour une croyance que $p$ de pouvoir être justifiée, alors que nous acceptons qu'il est possible d'avoir un état doxastique justifié de suspension de jugement, à savoir d'accepter et de tenir pour vrai le fait que $p$ ou non- $p$. Car puisque la suspension de jugement, comme attitude doxastique vis-à-vis une proposition, partage l'essentiel de sa nature avec la croyance ou la non-croyance (à savoir, d'être acceptée par le sujet et d'être dirigée vers la vérité), alors cet état peut être aussi justifié dans la mesure ou être justifié est la qualité d'un état doxastique quand il est approprié de l'avoir pour un sujet à un moment donné. Mais pourquoi permettre aux suspensions de jugements ce qu'on interdit aux croyances ou aux non-croyances? Il n'y a pas de différence de nature entre ces états doxastiques. Seul le contenu varie : $p$, non- $p$, $p$ ou non- $p$. Il semblerait alors que si le sceptique pyrrhonien nie la possibilité d'avoir des croyances justifiées, il doive par cohérence, pour 
aller jusqu'au bout de son pyrrhonisme, nier aussi la possibilité d'avoir un état doxastique justifié quelconque. Car affirmer que nous ne pouvons pas avoir de croyances justifiées, mais que nous pouvons avoir des suspensions de jugement justifiées mène au final à une sorte de paradoxe mooréen : ( $\neg$ Attitudedoxastique-justifiée-que- $p \wedge \neg$ Attitude-doxastique-justifiée-que-non- $p \wedge$ Attitudedoxastique-justifiée-que-p-ou-non-p). Il semble quelque peu étrange de dire : «Ma croyance que le Soleil tourne autour de la Terre n'est pas justifiée, et ma croyance que le Soleil ne tourne pas autour de la Terre n'est pas justifiée, mais par ailleurs ma croyance que le Soleil tourne autour de la Terre ou le Soleil ne tourne pas autour de la Terre est justifiée» ${ }^{15}$. Il paraît donc tout à fait arbitraire et étrange d'affirmer que nous n'acceptons pas $p$, ni non- $p$, mais que nous acceptons $p$ ou non- $p$. Pour suivre l'esprit pyrrhonien jusqu'au bout et pour éviter d'accorder un privilège arbitraire à une seule parmi les attitudes doxastiques, le pyrrhonien doit admettre que nous ne pouvons jamais avoir un état doxastique justifié, quel que soit le contenu de l'état en question ( $p$, non- $p$ ainsi que $p$ ou non- $p$ ). Mais en suivant cette route, nous aboutissons à une position dramatique et à tout jamais irréalisable : ce n'est plus une position précisant ou admettant les exigences ou les conditions que les croyances justifiées devraient satisfaire, mais une position de nihilisme doxastique - selon laquelle on ne doit avoir aucune attitude doxastique car aucune attitude doxastique n'est justifiable. Cela est d'une part impossible sur le plan cognitif et d'autre part contraire à toutes les intuitions (de sens commun comme celles des épistémologues). Même les sceptiques les plus durs accepteront qu'il est possible qu'un état doxastique soit plus justifié qu'un autre, étant donnée la plausibilité de données pertinentes. Un nihiliste doxastique ne peut pas accepter une telle intuition, car selon la conclusion nihiliste, il n'y a aucune différence entre les états doxastiques — ils sont tous injustifiés.

La deuxième objection est la suivante. Le sceptique pyrrhonien dira qu'il est possible de connaître que $p$, mais que nous ne pouvons jamais croire de manière justifiée que $p$ ou croire de manière justifiée que non- $p$, puisque la croyance justifiée est impossible. Cela se traduit : $(K(p) \wedge \neg B j(p))$ où $K(p)$ signifie : «connaître que $p$ », et $B j(p)$ : «être justifié à croire que $p$ ». Cela semble paradoxal, voire contradictoire. Or, il est intuitif et assez commun de penser, déjà depuis le Théétète de Platon, que la connaissance est au moins une croyance vraie justifiée. Donc si nous allions accepter le pyrrhonisme, nous devrions accepter aussi qu'il soit possible pour un sujet $S$ d'être dans un état inconsistant où $S$ connaît que $p$, mais ne croit pas que $p$ de façon justifiée. Si l'on accepte que la connaissance requiert (ou seulement implique) au moins la croyance vraie justifiée, alors une telle situation n'est pas possible à strictement parler.

Il nous semble, devant ces objections, que le scepticisme n'est pas une position qui peut répondre de manière satisfaisante aux questions de la croyance justifiée et de la possibilité de la connaissance.

D'un autre côté, un dogmatisme de style ancien ne semble pas satisfaisant non plus. Or, il ne peut pas répondre de manière appropriée aux arguments sceptiques et rendre compte des intuitions qui motivent les positions sceptiques. 
Il semble néanmoins qu'il existe une position promettant de réunir tous les avantages en ces matières. Face aux intuitions sceptiques et aux problèmes que ces positions rencontrent, le fidéisme se présente comme une option prometteuse, du moins pour une grande partie des penseurs de la fin de la Renaissance et du début de l'époque moderne ${ }^{16}$.

Mais comment le fidéisme peut-il répondre aux questions épistémiques et doxastiques? Premièrement, il est important de noter que dans la mesure où nous acceptons que les croyances religieuses puissent être discutées dans la même épistémologie générale que toute autre croyance, le fidéisme devient une position en épistémologie et non seulement en philosophie de la religion ou en théologie. Mais que dit le fidéisme?

\section{Fidéisme}

Certaines croyances ${ }^{17}$ n'ont pas besoin d'être justifiées par des raisons et/ou ne peuvent pas être justifiées par des raisons, mais sont justifiées par la foi seule.

En quoi cela répond-il à la question épistémique et à la question doxastique?

Tout d'abord, le fidéisme introduit une nouvelle distinction ${ }^{18}$ entre justification par la raison et justification par la foi. Grâce à cette distinction, une certaine sorte de fidéisme en tant que position épistémologique est compatible avec des intuitions sceptiques et évite pourtant les objections faites aux sceptiques. Le fidéiste va affirmer qu'il existe des croyances qui peuvent échapper au trilemme d'Agrippa et aux objections formulées à partir de scénarios sceptiques radicaux, tout en respectant les raisons sceptiques pour douter de nos capacités d'arriver aux croyances justifiées. Il existe une sorte de croyances qui échappe au problème de la justification parce qu'il existe une sorte de justification qui diffère de la justification présupposée par les arguments sceptiques. Il est important de dire aussi que les fidéistes peuvent soutenir ${ }^{19}$ que toute autre croyance qui en diffère n'est pas justifiée. La justification fidéiste ne présuppose pas l'usage (seul) des raisons ou des arguments. Cette justification est donnée par la foi (sans que nous ayons besoin des raisons). Par ailleurs, cette justification doit être ultime et infaillible pour pouvoir arrêter la régression à l'infini des justificateurs et pour bloquer le problème de la circularité.

La connaissance est aussi bien entendu possible pour le fidéiste.

La position fidéiste présente néanmoins un certain nombre de problèmes qui nous semblent impossibles à résoudre sans abandonner le fidéisme.

Le problème que nous voudrions soulever ici pose un dilemme au fidéiste. Le problème de ce dilemme est au final le problème concernant la notion de foi. En quoi «justifié par la foi» est-il vraiment différent de «justifié par la raison»? Il semble que seulement deux options soient possibles : soit cela ne diffère pas de manière radicale (foi et raison appartiennent au domaine théorique en tant qu'entités qui jouent le rôle de «justificateurs»), soit cela diffère, car l'un appartient au domaine théorique, alors que l'autre appartient au domaine pratique ou pragmatique. Nous sommes donc placés devant le dilemme suivant : 
1) Soit le fidéisme devient une sorte d'évidentialisme : la croyance d'une personne que $p$ est justifiée si et seulement si la personne dispose de raisons suffisantes en faveur de $p$. La justification par la foi et la justification par la raison consistent finalement à donner des raisons d'ordre théorique et dans ce cas, il n'y a pas vraiment de différence entre l'évidentialisme et le fidéisme ${ }^{20}$.

2) Soit le fidéisme devient une sorte de pragmatisme où la justification par la foi se réduit au final à donner des raisons non théoriques mais pratiques ou pragmatiques pour la croyance religieuse ${ }^{21,22}$. La position pragmatique semble néanmoins être problématique, et cela dans la mesure où il n'est pas clair qu'une position qui permet que des aspects pragmatiques interviennent dans l'attribution de la qualité d'être justifié ou dans l'attribution de la connaissance à un sujet puisse répondre aux questions épistémologiques et doxastiques. Il semble simplement qu'une telle théorie rate sa cible, puisque la croyance justifiée ou encore la connaissance appartiennent au domaine théorique (dans lequel les normes d'évaluation sont purement théoriques) et non au domaine pratique, et ne peuvent pas être dépendantes de considérations pragmatiques. Par exemple, le fait qu'une personne croie $p$ : qu'elle va être guérie de sa maladie parce qu'elle croit qu'elle va aller mieux ne peut pas être justifié, même si des raisons pragmatiques peuvent la motiver à le croire, comme par exemple le fait qu'il existe des cas où l'optimisme du malade joue un rôle dans la guérison d'une maladie.

En tout cas, pour les deux options, une croyance est justifiée sur la base de «quelque chose». Une croyance, selon le fidéiste, a besoin de «justificateurs» pour avoir la qualité d'être justifiée. Le choix fidéiste porte sur la spécificité de la nature de ces «justificateurs».

Il semble aussi que selon les deux options possibles, le fidéisme ne soit pas vraiment une position stable et indépendante, mais se réduise plutôt soit à l'évidentialisme, soit au pragmatisme concernant la connaissance et la croyance justifiée. Ainsi, le fidéisme se voit condamné soit à être réduit à l'un ou l'autre de ses rivaux, soit à être une position ambiguë contenant des éléments opposés.

Cela indique que le fidéisme n'est pas une position viable en épistémologie. Grâce à, et en même temps, malgré cela, historiquement le fidéisme a donné naissance à l'évidentialisme tolérant moderne. En ce sens, le fidéisme est la racine de l'évidentialisme moderne. Or, le fidéisme implique soit l'évidentialisme moderne, soit sa négation, et en tout cas son apparition.

\section{Évidentialisme : forme générale}

La croyance que $p$ de la personne $S$ est justifiée au moment $t$ si et seulement si la totalité des raisons probantes que possède $S$ au moment $t$ soutient $p^{23}$. 
Si nous acceptons la formulation tripartite de la connaissance qu'on trouve sous un certain aspect dans le Théétète de Platon, l'évidentialisme précise l'une des conditions qu'une croyance doit satisfaire pour constituer la connaissance ${ }^{24}$. La formulation tripartite de la connaissance énonce que la connaissance est la croyance vraie justifiée. Dans ce travail, nous ne chercherons pas à savoir si cette formulation de la connaissance correspond historiquement aux positions des philosophes antiques, médiévaux ou modernes. Pour notre intérêt, il nous suffit d'affirmer que l'une ou l'autre sorte d'évidentialisme était populaire dans l'époque moderne, ce qui ne veut pas dire que ce genre de position n'est pas compatible avec les théories de la connaissance médiévales ou antiques ${ }^{25}$.

Une fois que nous avons défini l'idée générale de l'évidentialisme, se pose la question des entités qui peuvent être considérées comme raisons probantes pour croire ou pour connaître une proposition. Nous n'aborderons pas cette question directement ici. Néanmoins, il semble que dans l'évidentialisme post-cartésien (y compris chez des auteurs comme John Locke), la conception internaliste de la raison probante était la plus populaire. Selon cette conception, ce qui constitue les raisons probantes appartient ontologiquement à la réalité mentale du sujet $^{26}$. On trouve aussi, toutefois, d'illustres exceptions à cette tendance. On ne peut omettre de mentionner l'exemple brillant de Thomas Reid. En combinant la thèse du réalisme direct de la perception avec une sorte d'évidentialisme, Reid accepta sûrement que ce qui constitue les raisons probantes n'est pas nécessairement de l'ordre du mental (au sens internaliste), mais qu'elles peuvent être constituées par des objets externes directement perçus.

L'évidentialisme moderne partage avec le scepticisme une prémisse de départ. Il s'agit de la prémisse qui exige des bases justifiantes pour qu'une connaissance ou une croyance soit justifiée. Et c'est le fidéisme qui permet à l'évidentialisme d'émerger, lequel évidentialisme précise la nature des bases exigées en attirant l'attention sur le fait que les croyances justifiées doivent reposer sur des raisons probantes.

L'évidentialisme propose certes une réponse aux questions épistémologiques que nous avons posées. Mais parvient-il à éviter les objections du scepticisme tout en expliquant les intuitions sceptiques? Ces réponses sont-elles adéquates?

Il semble que, seul, l'évidentialisme dans sa forme générale ne peut répondre à ces questions. Or l'évidentialisme ainsi conçu est vide ${ }^{27}$. Car dans son principe de base, l'évidentialisme ne dit rien concernant la façon dont on arrive à des croyances justifiées ou à la connaissance, ni concernant la nature des ces entités qui sont la base justifiante des croyances. Certes, il constitue une réponse possible aux deux questions qui nous intéressent. Mais si elle ne nous donne pas plus de détails, il est légitime alors de se demander si cette réponse est adéquate.

Une option d'inspiration moderne, remontant à Locke, Hume, Berkeley et d'autres, et largement héritée par les évidentialistes contemporains, consiste à préciser la nature des entités qui servent de raisons probantes en se tournant vers une conception internaliste. Il s'agit de la conception phénoménale des 
raisons probantes. Il est aisé de voir comment l'épistémologie internaliste de Descartes a contribué à l'émergence de cette conception. Mais cette conception est également redevable à des positions moins populaires, mais pourtant plus modérées - des positions à mi-chemin entre le scepticisme radical et «destructif» de La Mothe Le Vayer et le dogmatisme de Descartes. Ces positions sont celles de Pierre Gassendi, Francisco Sanchez ou encore Marin Marsenne (voir p. ex. Popkin, 1963), selon qui les sceptiques ont raison de nous faire douter de nos possibilités d'acquérir la connaissance en général de façon absolue, mais qui soutiennent néanmoins que nous pouvons connaître ce qui relève de domaines plus restreints. Il semblerait même qu'il revient à Gassendi d'avoir introduit pour la première fois la distinction entre certitude et connaissance, laquelle permet d'envisager la possibilité d'avoir une connaissance incertaine. Ce qui devient déterminant dans une telle perspective, ces sont les entités qui nous permettent d'avoir cette connaissance incertaine ou encore simplement une opinion justifiée. L'autre source de la conception phénoménale des raisons probantes se trouve dans les débats théologiques tenus aux $\mathrm{XVI}^{\mathrm{e}}$ et $\mathrm{XVII}{ }^{\mathrm{e}}$ siècles dans le contexte de la Réforme, et plus particulièrement dans les débats théologiques et politiques concernant la question de la tolérance (par exemple : William Chillingworth, John Tillotson). Il semblerait que la sécularisation de ces débats et leur transposition dans l'épistémologie ait servi d'inspiration à un certain évidentialisme et à la conception phénoménale des raisons probantes en mettant en évidence l'opposition suivante : (i) nous avons des positions non-conciliables; (ii) pourtant chacun doit pouvoir jouir de la tolérance religieuse/politique/ épistémologique; (iii) alors la seule façon de réconcilier, ou plus précisément, de ne pas juger irrationnelles les propositions (i) et (ii) est d'accepter que ce sur quoi repose le fait d'être «justifié» est propre à chacun individuellement et accessible seulement subjectivement.

Nous voudrions exprimer néanmoins des doutes concernant la conception phénoménale des raisons probantes. Cette conception semble finalement reposer sur une raison pragmatique - l'idée que l'on doive être tolérant vis-à-vis des convictions d'autrui.

Dans notre critique de la conception phénoménale des raisons probantes, nous suivons l'argument de Thomas Kelly selon lequel il n'y a pas de raisons convaincantes, à part les intuitions de penseurs modernes, pour accepter la conception phénoménale des raisons probantes (Kelly, 2008b). En effet, ni la théorie de la perception, ni l'usage ordinaire ou scientifique du concept de raison probante ne nous permettent de trancher en faveur de la conception phénoménale des raisons probantes.

Il est certes important de remarquer que la conception phénoménale des raisons probantes semble favoriser l'avènement de l'évidentialisme tolérant, ou le tournant tolérant pris par l'évidentialisme suite aux débats et spécifications découlant des défis sceptiques. Mais il est important de se rendre compte que l'évidentialisme peut être tolérant même sans adopter une conception phénoménale des raisons probantes ${ }^{28}$. 
Cela dit, nous croyons que c'est en grande partie à cause de l'apparition de la conception phénoménale des raisons probantes que l'évidentialisme tolérant a pris son essor. Si nous rejetons la conception phénoménale des raisons probantes, nous bloquons donc une des motivations pour l'acceptation de l'évidentialisme tolérant. Or, il semble un peu inutile de payer le prix de la conception phénoménale des raisons probantes tout en soutenant un évidentialisme de tolérance zéro. (Pourquoi admettre que les raisons probantes sont phénoménalement internes si nous rejetons la possibilité d'être trompé par elles? Intuitivement, présupposer la nature interne des raisons probantes semble une solution de secours face à la possibilité de l'erreur inconsciente (ou de l'illusion). Suivant cette solution, si l'on se trompe sans s'en rendre compte, c'est parce que les raisons probantes sont internes et accessibles à chacun personnellement, et ne sont pas nécessairement des indications objectives de la réalité). Par ailleurs, une telle combinaison des raisons probantes phénoménales avec l'évidentialisme de tolérance zéro semble très contre-intuitive, car cela impliquerait que tous ceux qui ont des raisons probantes concernant une question débattue partagent les mêmes raisons probantes internes (ou, du moins, des raisons probantes non-conflictuelles), car selon l'évidentialisme de tolérance zéro, des fausses propositions ne peuvent être soutenues par des raisons probantes. On observe pourtant des disputes et des désaccords dans lesquels chacun se réfère explicitement à ses propres raisons probantes, lesquelles sont très souvent conjointement incompatibles. Le rejet de la conception phénoménale des raisons probantes va donc de pair avec l'affirmation de l'évidentialisme de tolérance zéro et enlève une des motivations pour préférer l'évidentialisme tolérant.

Toutefois, il est nécessaire de donner un argument indépendant pour défendre l'évidentialisme de tolérance zéro. Notre argument est similaire à celui opposé par Williamson à la conception phénoménale des raisons probantes. En réalité, nous ne sommes pas nécessairement en mesure de connaître le statut de l'évaluation épistémique correcte de nos états doxastiques (il s'agit du principe de prudence doxastique), comme nous ne sommes pas nécessairement en mesure d'accéder à nos raisons probantes. Mais si nous ne savons pas nécessairement, alors face à l'erreur, à l'hallucination, à l'ignorance ou à la tromperie, nous ne devrions pas attribuer à nos croyances la propriété d'être «justifiées», même si les circonstances sont indistinctement similaires aux circonstances des situations dans lesquelles nous avons, ou avons eu, des croyances justifiées. Si une telle situation plutôt rare a effectivement lieu, et s'il n'existe aucun moyen de distinguer la croyance fausse en question de croyances vraies dans des contextes similaires, alors, certes, nous nous trompons si nous attribuons le statut de croyance «justifiée» à cette croyance sur la base de ce que nous croyons être les raisons probantes. Dans de telles situations — s'il n'y a pas de moyen de discerner si nous sommes dans un monde véridique ou dans un monde trompeur —, il nous faut pour ainsi dire «avaler la pilule», et éviter de se prononcer sur le statut de nos croyances. Néanmoins, pour la majorité des cas 
réels, il y aurait toujours une façon ou une autre de distinguer (le contexte trompeur de) la croyance fausse sur la base de la totalité des (de nos) raisons probantes. Et si, dans une situation où nous disposons de moyens permettant de distinguer le contexte de la croyance fausse du contexte de la croyance véridique, nous nous trompons sur le statut de nos croyances, alors cela relève de notre faute et de notre manque de discernement. Nous ne devrions pas nous tromper dans de telles situations, une bonne épistémologie ne devrait pas se tromper dans les situations où l'on peut discerner les contextes trompeurs des contextes véridiques. À l'opposé, si nous ne disposons pas des moyens permettant de distinguer les contextes trompeurs des contextes véridiques, alors il est impossible de connaître le statut exact de nos états doxastiques — il n'y a aucune façon de savoir si nos croyances correspondent, par exemple, à la réalité, ce qui est sûrement important pour connaître le statut de l'évaluation doxastique exacte des croyances en question. Dans ce cas, il vaut mieux s'abstenir de juger ces états doxastiques comme étant justifiés ou non-justifiés. En effet, s'il est impossible de distinguer des croyances vraies de croyances fausses, alors il est impossible de faire de l'épistémologie tout court. S'il s'avère que nous vivons dans un monde démoniaque dans lequel tout ce que nous croyons est faux, alors il est intuitivement normal de conclure que nous n'avons pas de moyen de faire de l'épistémologie et d'établir les statuts de l'évaluation doxastique. Pourtant, si nous pouvons trouver des moyens de discerner plutôt correctement dans la majorité des cas (sur la base de la totalité de nos raisons probantes, et pas seulement sur la base de raisons probantes occurrentes), alors il n'est pas nécessaire d'abandonner l'évidentialisme de tolérance zéro. Nous pouvons et devons simplement admettre que nous ne sommes pas nécessairement les meilleurs juges de nous-mêmes (et des autres) en ce qui concerne le statut de nos états doxastiques, plutôt que de promouvoir à tout prix une tolérance doxastique.

Ainsi, nous proposons plutôt d'adopter la conception de l'évidentialisme de tolérance zéro et, d'autre part, de rejeter la conception phénoménale des raisons probantes.

Nous pouvons maintenant nous tourner vers la deuxième question que nous avons annoncée, à savoir la question des conditions d'émergence de l'évidentialisme moderne. Nous y répondons en affirmant que l'évidentialisme moderne est possible et qu'il a émergé en grande partie grâce à la position fidéiste et en discussion avec elle, et plus particulièrement avec le fidéisme sceptique. Dans ce qui suit, nous nous attacherons à donner à cette thèse des bases plus fermes à partir de l'exemple de la controverse entre Pierre Bayle et le cartésien Jean-Pierre de Crousaz.

Notre thèse, répétons-le, veut que l'évidentialisme ait émergé en discussion avec le fidéisme, et plus précisément, en s'y opposant. L'évidentialisme primitif n'était pas nécessairement un évidéntialisme tolérant. C'est en réaction aux défis sceptiques des temps modernes et influencé par la conception phénoménale des raisons probantes que l'évidentialisme a pris, par précaution et par un 
trop grand souci de faire des concessions, le tournant tolérant. Nous pensons que cela n'était pas nécessaire.

Voyons un cas concret où est motivée l'émergence de l'évidentialisme.

\section{L'émergence de l'évidentialisme moderne}

Nous maintenons que c'est la distinction entre foi et raison qui a permis l'émergence de l'évidentialisme, et ce, en vertu du fait que cette distinction nous permet de faire la spécification, cruciale pour l'évidentialisme, entre les raisons probantes et tout ce qui n'en est pas.

L'importance de cette distinction est grande dans les travaux de Pierre Bayle, mais aussi dans la réponse que fait Jean-Pierre de Crousaz à son fidéisme sceptique.

\subsection{Le scepticisme fidéiste de Pierre Bayle}

La philosophie de Pierre Bayle (1647-1706) fut une de sources implicites du programme philosophique des Lumières (Lennon et Hickson, 2012). D’après certains, Bayle aurait même pu tirer les mêmes conclusions, ou plutôt présenter les mêmes positions que celles défendues plus tard par David Hume (Milton, 1987). Ce qui lui a manqué pour y parvenir, selon certains, serait la systématicité de la pensée.

On ne peut que sous-estimer son importance dans l'histoire des idées. Son rôle fut prédominant non seulement dans l'élaboration d'une position scepticofidéiste, dans la diffusion, la prise au sérieux et la popularisation du scepticisme (jusqu'à Hume, selon Popkin, 1963 et 2003), mais aussi dans d'autres domaines de la philosophie tels que la philosophie politique, spécialement en ce qui concerne la question de la tolérance religieuse et politique.

Né en France près de Toulouse, Bayle a étudié la philosophie de Descartes à Genève.

En matière d'épistémologie, Pierre Bayle a soutenu ce qu'on appelle parfois le «super-scepticisme» (Popkin, 2003). Son scepticisme a consisté à remettre en question tout. Il proposait des arguments contre toute théorie et son contraire. Sa position était méthodologique et consistait à appliquer le raisonnement jusqu'à l'élimination de toute erreur.

Pierre Bayle a sans doute contribué à la popularisation du scepticisme pyrrhonien, même si des découvertes et des interprétations importantes avaient déjà circulé auparavant. Cicéron et le scepticisme académique ont été beaucoup plus connus grâce à divers facteurs, notamment l'intérêt de saint Augustin pour les académiciens, dans la mesure où il propose une réfutation de ce scepticisme. Toutefois, le scepticisme de Bayle lui-même serait non pas celui des pyrrhoniens, mais serait plutôt de type académicien. Il serait plutôt de l'ordre des doutes méthodologiques (Lennon et Hickson, 2012).

Il s'agit pourtant d'un scepticisme bien spécifique : d'un scepticisme laissant place à la foi en matière religieuse. Si Hume a pu écrire : «notre scepticisme laisse la place à la foi chrétienne», cette affirmation ne permet pas de décrire sa foi, 
pour des raisons de compatibilité de ses positions philosophiques et religieuses. La formule paraît cependant parfaitement adaptée pour exprimer la position de Pierre Bayle.

Pourtant, je voudrais remettre en question la thèse selon laquelle ce qui distingue Hume de ses prédécesseurs est sa systématicité et surtout sa liberté concernant la religion, dont ses prédécesseurs ne jouissaient pas entièrement selon certains (voir p. ex. Hacking, 1984 [1975]; Milton, 1987).

Je m'oppose à cette thèse à partir de deux arguments, l'un négatif, l'autre positif - et cela m'amène à proposer une autre thèse. 1) Si l'on poursuit jusqu'au bout cette idée, nous arrivons à la thèse voulant que les arguments philosophiques ne s'appliquent pas, ne fonctionnent pas pareillement chez les théistes et chez les athées. Or, cela est impliqué lorsqu'on admet qu'il fallait être libre de religion pour comprendre et affirmer tel que Hume l'a fait. Le seul sens dans lequel nous pouvons comprendre «être libre de religion» sans tomber dans l'absurdité est «ne pas être théiste». On ne peut soutenir qu'être «libre de religion» était seulement une question de contexte philosophique. D'une part, au Moyen Âge et plus tard, mais en tous cas avant Hume, il y eut des lieux et des temps «extérieurement» libres, autrement dit sans contrainte politique. D'autre part, il y eut des philosophes qui ne considéraient pas les contraintes «externes» comme des contraintes véritables et qui ont continué à annoncer leurs théories, de Pierre Abélard à Pierre Bayle lui-même, qui n'avait pas peur de dire exactement ce qu'il avait à dire même si cela impliquait des contraintes pour lui ou pour sa famille (Lennon, 2008). 2) La raison pour laquelle Bayle n’est pas «devenu» Hume n'est pas sa religion, mais plutôt son fidéisme.

\subsection{L'évidentialisme de Jean-Pierre de Crousaz}

Comme cela arrive en philosophie, les thèses de Pierre Bayle ont été critiquées. Jean-Pierre de Crousaz fut l'un de ses critiques inépuisables en matière de philosophie de la connaissance. Étant encore jeune, de Crousaz avait rencontré Bayle à Paris. Il était en désaccord absolu avec sa position.

Jean-Pierre de Crousaz (1663-1750), philosophe suisse romand né à Lausanne, appartient à la tradition cartésienne dans son déclin. Sa contribution n'est pas de l'ordre des changements de paradigme. Néanmoins, il nous semble qu'il a contribué dans une certaine mesure à la popularisation de l'évidentialisme.

Genève et la Suisse romande ont été des lieux centraux pour l'émergence du scepticisme moderne. En premier lieu, c'est à Genève que Henri Estienne a imprimé en 1562 sa nouvelle traduction latine des œuvres de Sextus Empiricus, réintroduisant ainsi le pyrrhonisme de Sextus sur la scène philosophique. Certains auteurs (Popkin, 2003) affirment même que cette nouvelle édition, ainsi que celle de 1569 , ont joué un rôle décisif pour le développement du scepticisme moderne et sa distinction par rapport aux problèmes sceptiques médiévaux. Cela peut toutefois être discuté. Il semblerait que les mêmes problèmes aient été discutés par les penseurs médiévaux sans qu'ils aient eu facilement accès aux œuvres pyrrhoniennes de Sextus (Perler, 2004). Quoiqu'il en soit, 
Genève a été le lieu à partir duquel les textes de Sextus ont recommencé à circuler. C'est aussi à Genève que le jeune Pierre Bayle a étudié la philosophie de Descartes. Jean-Pierre de Crousaz, son adversaire acharné, a également étudié à Genève. À cela s'ajoute le rôle qu'ont joué le calvinisme et le protestantisme en général dans les débats sur les statuts de nos croyances et de nos connaissances, débats concernant au premier chef le scepticisme et le fidéisme.

Les réponses de Jean-Pierre de Crousaz à la position de Pierre Bayle n'ont pas produit un grand mouvement de réaction contre son scepticisme. Toutefois, de Crousaz a proposé quelques arguments dignes d'attention ${ }^{29}$.

Dans Examen du pyrrhonisme ancien et moderne (2003 [1733]), Jean-Pierre de Crousaz s'oppose en détail (en deux tomes totalisant 2500 pages!) à deux positions sceptiques. D'une part, il combat le pyrrhonisme de Sextus Empiricus. D'autre part, il s'attaque à la doctrine de Pierre Bayle. L'approche de de Crousaz peut paraître par moments déplacée, à cause de l'usage fréquent des arguments de type ad hominem et des répétitions constantes de ses idées, qui ne facilitent pas la lecture.

Pourtant, certains passages de l'ouvrage présentent un véritable intérêt pour notre hypothèse. Nous présenterons deux aspects de l'argumentation de de Crousaz qui semblent soutenir notre thèse.

Jean-Pierre de Crousaz défend d'une part la compatibilité de la doctrine chrétienne avec la raison — plus précisément, c'est une thèse encore plus forte que de Crousaz semble défendre : la nécessité d'avoir des preuves avant d'adhérer à la foi chrétienne. D'autre part, de Crousaz fait le constat que nous avons seulement deux possibilités quand nous nous engageons dans une discussion philosophique : soit suivre la raison (proposer des raisons pour soutenir la vérité d'une thèse), soit accepter qu'il existe quelque chose de l'ordre non-théorique qui peut avoir une plus grande valeur que la recherche de la vérité.

C'est dans la troisième partie de son ouvrage que Jean-Pierre de Crousaz s'attaque à Pierre Bayle à partir de questions très différentes. Parmi les aspects de la pensée de Bayle que de Crousaz critique se trouve la question de la relation entre la foi et la raison ${ }^{30}$. Tout d'abord, de Crousaz reprend l'objection de Bayle selon laquelle il ne faut jamais se baser sur notre raison pour comprendre ce que la foi nous enseigne. Selon Bayle (d'après de Crousaz) :

Il ne faut jamais accorder, dit-il, cette condition que si le sens litéral d'un passage de l'Ecriture renferme des Dogmes inconcevables, et combatus par les Maximes les plus évidentes des Logiciens, et des Métaphysiciens, il sera déclaré faux et que la Raison, la Philosophie, la Lumiére Naturelle, seront la régle que l'on suivra pour choisir une certaine interprétation de l'Ecriture préférablement à toute autre (de Crousaz, 2003 [1733], p. 872).

S'il faut éviter de se servir de la raison pour distinguer, dans les cas complexes, quel est le sens de la doctrine chrétienne contenue dans la Bible, alors on peut aussi accepter qu'il ne faille pas se fonder sur notre raison tout court pour 
comprendre les vérités de la foi. C'est ce que Bayle semble dire quand il imagine l'opposition entre saint Paul et les philosophes grecs sur les vérités de la foi. Bayle conclut ainsi que l'apôtre aurait pu dès le départ (et ce serait donc, selon Bayle, une bonne chose) mettre en évidence la différence entre l'acceptation des vérités de l'Évangile sur la base de raisonnements et sur la base de la foi seule :

[...] après qu'il leur auroit déclaré le fondement de sa créance, il auroit pû leur opposer cette barriére, que ces dogmes étoient inconnus à la Raison qu'ils avoient été révélés de Dieu, et qu'il falloit les croire sans les comprendre (de Crousaz, 2003 [1733], p. 873).

Avant de poursuivre avec l'objection de Jean-Pierre de Crousaz, il est important de noter la distinction, non-explicitée, entre le raisonnement (l'activité de la faculté de raison) et les raisons en tant que preuves (les objets des raisonnements, à partir desquels la raison tire des conclusions). Ainsi, quand un auteur dit par exemple que la raison et la foi doivent être compatibles, cela implique certes que l'on doive s'engager dans l'activité de raisonnement, mais cela implique aussi que nous devons avoir des raisons — des preuves à partir desquelles on tire des conséquences. Cela dit, l'usage que Pierre Bayle fait des ces termes semble impliquer un principe encore plus fort, à savoir que pour avoir la foi, il ne faut même pas utiliser la faculté de raison, et non simplement que nous n'avons pas de preuves (ou du moins que nous n'avons pas une certaine sorte de preuves) à partir desquelles on pourrait inférer les propositions concernant la foi.

Quelle est la réponse de Jean-Pierre de Crousaz à cette position? De Crousaz s'oppose à cette approche de Bayle. Tout d'abord, il semble remettre en question l'interprétation que fait Bayle de l'attitude possible de saint Paul. De Crousaz conteste l'idée voulant que saint Paul trouverait normale la situation dans laquelle on accepte la foi sans faire l'usage de notre raison : face aux contradictions dans la doctrine,

[...] St. Paul auroit-il dit; N'importe, croire de tels sentiments, sans aucun doute, c'est-le mérite de la Foi? Mais quand les Philosophes auroient répliqué, le sens commun s'y oppose; St. Paul auroit-il ajouté, il ne faut point l'écouter, les Lumières de la Raison sont toutes incertaines et trompeuses? Alors les Philosophes n'auroient pas manqué de dire; Que venés vous donc faire ici? Nous ne saurions nous assurer que vous nous parlés, et quand nous n'aurions aucun doute sur ce point, nous ne saurions nous assurer si nous comprenons le sens de vos paroles, et si, lorsque nous nous flatterions d'être vos disciples, nous ne croions pas peut-être, tout le contraire de ce que vous pensés (de Crousaz, 2003 [1733], p. 874).

En fait, Jean-Pierre de Crousaz est en train de mettre de l'avant l'argument de l'impossibilité de comprendre le message de la bonne nouvelle sans l'usage 
de la raison. La raison (ici en tant qu'activité du raisonnement) n'est donc pas simplement compatible avec la foi, mais elle lui est nécessaire. L'acquisition même de la foi dépend ainsi selon de Crousaz de la raison. Si nous ne raisonnions pas, nous ne pourrions pas comprendre ce que signifie le contenu de notre foi. Ici, une objection naturelle serait la suivante : mais à quel niveau de compréhension devons-nous nous arrêter pour avoir une foi authentique? Quel est le niveau suffisant? Seuls les théologiens, peut-être, peuvent comprendre certains articles de la foi, on acceptera pourtant de dire que même une personne laïque croit en l'article de la foi en question. Pour répondre à cela, on pourrait dire que Jean-Pierre de Crousaz voulait probablement établir seulement la nécessité d'un niveau minimal : c'est-à-dire qu'un niveau minimal de compréhension (résultant de l'activité de raisonnement) est nécessaire pour la possession de la foi. Si nous n'utilisons pas du tout notre raison, nous ne pouvons acquérir ce niveau minimal. En ce sens, le raisonnement est toujours nécessaire à la foi. Mais le raisonnement est-il seul nécessaire à la foi selon Jean-Pierre de Crousaz? Ne faut-t-il pas en avoir aussi les preuves?

Effectivement Jean-Pierre de Crousaz défend aussi cette deuxième thèse de la dépendance entre la foi et la raison. C'est surtout cette thèse qui vient confirmer notre hypothèse que le fidéisme sceptique a joué un rôle déterminant dans l'émergence de l'évidentialisme moderne.

Un homme qui se met, à tout hazard, dans l'esprit que les Livres que nous appellons le Vieux et le Nouveau Testament, sont Divins, et cela sans éxamen et sans preuve, ou qui se le persuade uniquement parce qu'il trouve, cette persuasion établie dans son Païs, est-il louable, fait-il son devoir? et s'il est capable de raisonner, et de s'assurer de la Divinité de ces Saints Livres par un éxamen sincére, et par des preuves dignes d'eux, est-il pardonnable de ne le pas faire? De son côté il ne fait que ce que font [...] tous ceux qui reçoivent pour Divin ce qui ne l'est pas. S'il se trompe ou s'il ne se trompe pas, c'est le hazard qui en décide; Il faut donc s'être assuré que la voie du Raisonnement peut conduire à quelque certitude, et que le Pyrrhonisme est une éxtravagance; sans quoi on n'aura jamais sur la Vérité de la Révélation, sur laquelle on fonde sa Foi, que des preuves douteuses (de Crousaz 2003 [1733], p. 900).

Selon Jean-Pierre de Crousaz, nous avons besoin de preuves pour être raisonnables dans notre foi. Si nous maintenons que nous n'avons pas besoin de preuves, alors nous ne pourrons pas faire la distinction entre quelqu'un qui forme une croyance de façon déraisonnable et quelqu'un qui forme une croyance de façon raisonnable. Une version de l'évidentialisme moderne est ainsi défendue. De plus, Jean-Pierre de Crousaz semble discuter déjà de la nature même de la justification. Il semble définir la conception de la justification en termes de blâme et de louange, et cela, bien avant les conceptions déontologiques de la justification, et bien avant William Kingdon Clifford, Roderick Chisholm et d'autres. 
Un autre point qui semble soutenir notre hypothèse est le suivant : dans son Examen du pyrrhonisme ancien et moderne, de Crousaz accuse Bayle de ne pas suivre la raison, ou plus encore, d'être vulnérable à l'objection de la régression à l'infini (v. p. ex. de Crousaz, 2003 [1733], p. 907). Puisqu'il accepte la foi chrétienne, bien que cela soit seulement sous la forme fidéiste, affirme de Crousaz, Bayle devrait baser sa foi sur quelque chose, notamment sur les témoignages des apôtres ou sur la Bible. S'il croit aux vérités de la foi, c'est à cause de quelque chose. Pourtant, si Bayle rejette la possibilité de justification par les raisons (quelles qu'elles soient), cela réduit sa position à la contradiction : Bayle ne peut pas croire et même temps il peut croire - Bayle croit, et croit sur la base de témoignages, et en même temps ne doit pas croire, car aucune raison ne peut justifier une croyance.

De Crousaz accuse Bayle de ne pas être ce qu'on appelle un évidentialiste des Lumières et d'être plutôt pragmatiste en ce qui concerne la vérité. Mais cela est contraire à l'esprit de l'évidentialisme.

Notre hypothèse se voit donc confirmée : de Crousaz était évidentialiste. L'émergence de l'évidentialisme des Lumières est due à ce genre de discussions plus qu'aux doutes sceptiques.

\section{Conclusion}

En guise de conclusion, nous pouvons donc affirmer que ni le scepticisme, ni le fidéisme ne nous permettent de répondre de manière satisfaisante aux questions fondamentales de l'épistémologie.

L'évidentialisme amélioré est la position la plus adaptée pour répondre à la question de l'épistémologie. Nous soutenons aussi que l'évidentialisme est apparu grâce au fidéisme chrétien du début de l'époque moderne ${ }^{31}$.

\section{Notes}

1 Dans cet article, nous appelons «croyance» une attitude propositionnelle du sujet $S$ envers une proposition $p$ telle que $S$ accepte et tient pour vraie la proposition $p$. Les croyances diffèrent de simples pensées par le fait que les pensées n'ont pas la caractéristique d'être dirigées vers la vérité. Une personne peut, sans aucune inconsistance ou sans être paradoxale, avoir une pensée tout en sachant que le contenu de cette pensée est faux, tandis que cela n'est pas possible pour les croyances (cette caractéristique de la croyance est mise en lumière notamment par le paradoxe de Moore. Cf. «Moore's Paradox», dans G. E. Moore, 1993, p. 207-212). Il n'est pas rare de voir utilisés les mots «opinion» et «croyance» dans un sens proche. Nous acceptons que l'opinion (doxa) soit un synonyme de croyance. Par «état doxastique», nous entendons : les croyances, les non-croyances et les suspensions de jugement. Concernant le contenu des croyances, nous assumons dans ce travail que le contenu des croyances ne permet pas de distinguer entre différents types des croyances. Ainsi, nous supposons qu'il s'agit d'un même type d'attitude indifféremment du contenu d'une croyance : les croyances ordinaires appartiennent au même type d'attitude que les croyances religieuses, par exemple, ou que les croyances scientifiques. 
2 Par «raisonnable», nous désignons la qualité d'une croyance d'une personne quand il est approprié pour cette personne d'avoir cette croyance. La qualité «raisonnable» sera donc prêtée ici aux croyances effectivement crues, et non aux croyances potentielles, ou aux attitudes qu'il serait approprié d'avoir dans tel contexte. Pour des raisons de clarté, nous assumons dans ce travail que «croyance raisonnable» est synonyme de «croyance rationnelle» ou encore de «croyance justifiée». Par souci d'être fidèle à l'usage le plus répandu dans la littérature consacrée à l'épistémologie, nous utiliserons en règle générale le terme «justifié».

3 Voir par exemple, pour la définition de l'épistémologie en ces termes, Stroud, 2005.

4 Selon Katja Vogt, les questions de la croyance justifiée, de la certitude, du doute et même de la connaissance, contrairement aux questions concernant la croyance, la suspension du jugement ou encore le critère de la vérité, ne faisaient pas partie des centres d'intérêt des philosophes anciens, mais n'apparaissent à proprement parler qu'en philosophie moderne (Vogt, 2011).

5 Voir à ce sujet Perler, 2004; Lagerlund, 2010. Toutefois, c'est une autre question que de savoir combien le scepticisme ancien est proche du scepticisme moderne (voir entre autres, pour des positions divergentes : Annas et Barnes, 1985; Bailey, 2002; Menn, 1998; Carriero, 2009; Williams, 2010; Vogt, 2011; Burnyeat, 1980; et enfin Fine, 2000). Ainsi, par exemple, Gail Fine défend la thèse voulant que le scepticisme cartésien ait été beaucoup plus proche du scepticisme ancien qu'on ne l'avait pensé (Fine, 2000).

6 Le français contemporain ne nous permet pas d'utiliser le terme «évidence» pour notre description de l'évidentialisme. Des épistémologues français ont souvent remarqué cette difficulté (Dutant et Engel, 2005; Engel, 2006; Jacob, 1980). Le terme anglais «evidence» conviendra mieux pour notre formulation. Nous ne ferons malheureusement pas ici une étude concernant cette différence d'usage. Un tel travail sortirait de notre champ d'investigation. Nous précisons néanmoins que nous allons utiliser au long de ce travail les termes «évidence» et «raison probante» de manière interchangeable, pour désigner ce que nous croyons être le terme clé dans le principe évidentialiste : cette entité qui permet à l'agent épistémique d'avoir une croyance justifiée ou une connaissance.

$7 \mathrm{La}$ formulation présente est redevable pour l'essentiel à la formulation des évidentialistes contemporains - par exemple Conee et Feldman, 2004. On peut associer l'évidentialisme moderne (dans sa forme «tolérante») à John Locke (par exemple dans An Essay Concerning Human Understanding), David Hume (par exemple dans Enquiries Concerning Human Understanding and Concerning the Principles of Morals) et George Berkeley. On peut probablement trouver (au moins les racines de) cet évidentialisme tolérant même plus tôt, par exemple chez Richard Whately, William Paley et d'autres auteurs, dans le contexte de disputes religieuses entre protestants et catholiques (voir Sweet, 1999). Le questionnement moderne concernant le lien entre la connaissance et la certitude, ainsi que la séparation entre les deux, est certainement aussi un point qui permettrait de retracer les origines de l'évidentialisme tolérant (voir par exemple Leeuwen, 1970). 
8 Dans ce travail, je ne présuppose aucune position concernant ce que sont les propositions. J'accepte simplement que le contenu des croyances soit propositionnel. Plusieurs positions existent concernant la nature des propositions : certains les définissent comme des mondes possibles, d'autres croient qu'elles sont ce qui correspond aux états de choses.

9 Pour la formulation de cette distinction, cf. Klein, 2002.

10 Une affirmation sceptique concernant l'impossibilité de la connaissance semble impliquer que nous pouvons connaître au moins cette vérité sceptique. Le scepticisme académique ne peut donc pas être vrai à propos de toutes les propositions, car il $\mathrm{y}$ en a au moins une qui peut être connue - le fait de ne pas pouvoir connaître.

11 D'après les arguments sceptiques attribués au philosophe antique Agrippa.

12 Certains auteurs contemporains ont argumenté en faveur de la compatibilité de certaines de ces options face à la problématique de la justification des croyances. Voir Haack, 1999; Sosa, 1980; mais aussi Conee, 1988.

13 C'est l'intuition principale fondant les doutes radicaux présents dans les Méditations Métaphysiques, mais c'est aussi l'intuition d'un certain nombre des penseurs du Moyen Âge qui ont réfléchi au problème sceptique, comme Guillaume Crathorn ou encore Al-Ghazâlî. Il est argumenté que les scénarios sceptiques, ou la possibilité de tels scénarios, émergent en grande partie au Moyen Âge à cause de l'ampleur que prend la discussion sur la toute-puissance divine. Si Dieu est tout-puissant, il existe la possibilité qu'il nous trompe sur ce que nous pensons, et cela, peut-être même de manière radicale. Certains argumentent que les racines de ces scénarios sceptiques remontent au moins aux condamnations de Paris de 1277 et à des travaux d'Henri de Gand sur la condamnation comme hérétiques de ceux qui nient la toutepuissance Divine (voir Bolyard, 2009).

14 Certains argumentent que le concept même de croyance justifiée n'a aucun sens pour le scepticisme ancien (Vogt, 2011). D'autres insistent sur les similitudes entre le scepticisme cartésien et le scepticisme ancien (Fine, 2000).

$15 \mathrm{~J}$ 'assume ici que la suspension de jugement à propos de $p$ et non- $p$ est égale à la croyance que $p$ ou non- $p$. Il me semble que c'est l'interprétation de la suspension de jugement la plus correcte et pertinente pour l'épistémologie de la croyance. Si l'on n'accepte pas cette interprétation, alors on présuppose que la suspension de jugement n'est pas un état doxastique et donc n'est pas soumise aux mêmes normes épistémiques d'évaluation doxastique, mais s'approche plutôt des pensées, du fait de deviner («guessing»), autrement dit d'attitudes qui ne visent pas nécessairement la vérité.

16 Ceux qui se dénommaient «les chrétiens sceptiques», comme Pierre Charron, Pierre Huet, Pierre Gassendi ou même Michel de Montaigne lui-même, peuvent être appelés par de nombreux aspects des fidéistes.

17 Notamment les croyances religieuses.

18 Nous soutenons dans la suite de notre travail que cette distinction permet l'émergence de l'évidentialisme moderne.

19 Et souvent les fidéistes modernes le maintiennent, notamment pour arriver à un nouvel argument pour la justification de croyances religieuses. 
20 Les fidéistes contemporains semblent aller dans cette direction quand ils élaborent le point de vue du fidéisme rationnel (voir Evans, 1998; Bishop, 2007).

21 Certains fidéistes ont pris cette voie, notamment Blaise Pascal, qui épouse une sorte de fidéisme selon lequel ce sont les raisons pratiques ou pragmatiques qui nous amènent à avoir certaines croyances justifiées (le pari de Pascal). On pourrait aussi interpréter la position de Pierre Bayle comme appartenant à ce groupe. Or, il accepte qu'il y ait des raisons probantes morales pour accepter la croyance religieuse.

22 Il est aussi intéressant de noter que les opposants contemporains les plus éloignés de l'évidentialisme sont des philosophes qui acceptent que des aspects pragmatiques interviennent de manière déterminante dans nos attributions de connaissances et de croyances justifiées. Voir par exemple Fantl et McGrath (2009), Keith DeRose $(1992,1996)$ et la question de l'empiètement pragmatique.

23 Nous pouvons trouver cette sorte d'évidentialisme et sa défense en détails chez certains philosophes contemporains : le cas paradigmatique est celui de Conee et Feldman, 2004. On peut en trouver une formulation classique chez Clifford, notamment dans «The Ethics of Belief», 1999.

24 La définition néanmoins doit être améliorée, soit pour rendre la théorie de la connaissance faillibiliste, soit pour rendre l'évidentialisme infaillibiliste. Or, la condition de temporalité dans la formulation de l'évidentialisme fait de cet évidentialisme un évidentialisme faillibiliste.

25 Pour une thèse selon laquelle l'apparition du concept de raison probante («evidence») interne est décisif dans le saut qualitatif entre David Hume et ses prédécesseurs concernant l'induction, voir Hacking, 1984. Voir Milton, 1987 et Blackburn, 1976 pour une critique de cette position.

26 Thomas Kelly expose les racines modernes de la conception dite phénoménale des raisons probantes, soit la conception affirmant que les raisons probantes sont des états mentaux non-factifs (les états mentaux factifs impliquent la vérité de leur contenu) (Kelly, 2008b).

27 Juan Comesaña affirme que l'évidentialisme tout seul est vide, comme le fiabilisme est aveugle (le fiabilisme est une autre position contemporaine en épistémologie selon laquelle tout ce qui est nécessaire pour avoir une croyance justifiée est qu'elle soit produite par un processus fiable qui produit de manière globale plutôt des croyances vraies). Une unification de deux théories apparaît à Comesaña, pour diverses raisons, une option nécessaire (Comesaña, 2010).

28 Par exemple, selon Timothy Williamson, nous devons abandonner la conception phénoménale des raisons probantes parce que nous ne sommes pas nécessairement en mesure d'accéder à ce qui fait partie de nos raisons probantes, mais il considère néanmoins que les raisons probantes peuvent conférer de la justification, du soutien à une proposition fausse - ce qui revient finalement à adhérer à un évidentialisme tolérant (Williamson, 2000).

29 Nous basons surtout notre analyse sur son principal ouvrage en théorie de la connaissance, Examen du pyrrhonisme ancien et moderne (de Crousaz, 2003 [1733]).

30 Nous nous concentrons surtout sur la fin de la section 2 de la partie 3 (de Crousaz, 2003 [1733], p. 847-915). 
31 Je voudrais remercier Pascal Engel et Marie Robert pour les discussions et les commentaires concernant les versions antérieures du présent article. Le travail qui a mené vers la présente publication était soutenu par le projet no $100018 \_131794$ «Connaissance, preuves et pratique», du Fonds National Suisse.

\section{Références bibliographiques}

Amesbury, Richard

2012 «Fideism», dans Edward N. Zalta, dir., The Stanford Encyclopedia of Philosophy, <http://plato.stanford.edu/archives/win2012/entries/fideism/> (édition hiver 2012).

Annas, Julia et Jonathan Barnes

1985 The Modes of Scepticism: Ancient Texts and Modern Interpretations, Cambridge, Cambridge University Press.

Bailey, Alan

2002 Sextus Empiricus and Pyrrhonean Scepticism, Oxford, Oxford University Press.

Bates, David

1996 «The Epistemology of Error in Late Enlightenment France», EighteenthCentury Studies, vol. 29, n 3, p. 307-327.

Bett, Richard

2010 The Cambridge Companion to Ancient Scepticism, Cambridge, Cambridge University Press.

Bishop, John

2007 Believing by Faith: An Essay in the Epistemology and Ethics of Religious Belief, Oxford, Oxford University Press.

Blackburn, Simon

1976 «The Emergence of Probability by Ian Hacking [compte rendu]», Philosophy, vol. 51, no 198, p. 476-480.

Bolyard, Charles

2009 «Medieval skepticism», dans Edward N. Zalta, dir., The Stanford Encyclopedia of Philosophy, <http://plato.stanford.edu/archives/spr2009/ entries/skepticism-medieval/>.

Burnyeat, Myles

1980 «Can the Sceptic Live His Scepticism?», dans Malcolm Schofield, Myles Burnyeat et Jonathan Barnes, dir., Doubt and Dogmatism: Studies in Hellenistic Epistemology, Oxford, Clarendon Press/New York (NY), Oxford University Press, p. 25-57.

Carriero, John Peter

2009 Between Two Worlds: A Reading of Descartes's Meditations, Princeton (NJ), Princeton University Press.

Clifford, William Kingdon

1999 The Ethics of Belief and Other Essays, Amherst (NY), Prometheus Books. Comesaña, Juan

2010 «Evidentialist Reliabilism», Nous, vol. 44, n 4, p. 571-600. 
Conee, Earl

1988 «The Basic Nature of Epistemic Justification», The Monist, vol. 71, n 3, p. 389-404.

Conee, Earl et Richard Feldman

2004 Evidentialism, Oxford, Oxford University Press. de Crousaz, Jean-Pierre

2003 Examen du pyrrhonisme ancien et moderne [1733], 2 tomes, Paris, Fayard.

2004 «Lettres sur 1'éducation - Examen du pyrrhonisme ancien et moderne», dans André Bandelier et Sébastien Charles, «Jean-Pierre de Crousaz: philosophe lausannois du siècle des Lumières. (Avec deux inédits de Crousaz)», Revue de Théologie et de Philosophie, vol.136, $\mathrm{n}^{\mathrm{o}} 1$, p. 71-79.

de La Harpe, Jacqueline Ellen Violette

1955 Jean-Pierre de Crousaz (1663-1750) et le conflit des idées au siècle des Lumières, Berkeley (CA), University of California Press.

DeRose, Keith

1992 «Contextualism and Knowledge Attributions», Philosophy and Phenomenological Research, vol. 52, no 4, p. 913-929.

1996 «Knowledge, Assertion and Lotteries», Australasian Journal of Philosophy, vol. 74, no 4, p. 568-580.

Engel, Pascal

2006 Va Savoir! De la connaissance en général, Paris, Hermann.

Engel, Pascal et Julien Dutant, dir.

2005 Philosophie de la connaissance. Croyance, connaissance, justification, Paris, Vrin.

Evans, C. Stephen

1998 Faith Beyond Reason, Édimbourg, Edinburgh University Press.

Fairlamb, Horace

2010 «Sanctifying Evidentialism», Religious Studies, vol. 46, n 1, p. 61-76.

Fantl, Jeremy et Matthew McGrath

2009 Knowledge in an Uncertain World, Oxford/New York (NY), Oxford University Press.

Fine, Gail

2000 «Descartes and Ancient Skepticism: Reheated Cabbage?», The Philosophical Review, vol. 109, no 2, p. 195-234.

Haack, Susan

1999 «A Foundherentist Theory of Empirical Justification», dans Louis Pojman, dir., The Theory of Knowledge: Classical and Contemporary Readings, 2e éd., Belmont (CA), Wadsworth, p. 283-293.

Hacking, Ian

1984 The Emergence of Probability: a Philosophical Study of Early Ideas About Probability, Induction and Statistical Inference [1975], Cambridge (NY), Cambridge University Press. 
Holyer, Robert

1989 «Scepticism, Evidentialism and the Party Argument: A Pascalian Perspective», Religious Studies, vol. 25, n 2, p. 191-208.

Iltis, Carolyn

1973 «The Decline of Cartesianism in Mechanics: The Leibnizian-Cartesian Debates», Isis, vol. 64, no 3, p. 356-373.

Jacob, Pierre

1980 L'empirisme logique : ses antécédents, ses critiques, Paris, Minuit.

Kelly, Thomas

2008a «Evidence», dans Edward N. Zalta, dir., The Stanford Encyclopedia of Philosophy, <http://plato.stanford.edu/archives/fall2008/entries/ evidence/> (édition automne 2008).

2008b «Evidence: Fundamental Concepts and the Phenomenal Conception», Philosophy Compass, vol. 3, nº 5, p. 933-955.

Winkler, Kenneth, P.

2004 «Berkeley, Pyrrhonism, and the Theaetetus», dans Walter SinnottArmstrong, dir., Pyrrhonian Skepticism, Oxford, Oxford University Press, p. 40-67.

Klein, Peter

2002 «Skepticism», dans Paul K. Moser, dir., The Oxford Handbook of Epistemology, Oxford, Oxford University Press, p. 335-360.

2011 «Skepticism», dans Edward N. Zalta, dir., The Stanford Encyclopedia of Philosophy, <http://plato.stanford.edu/archives/sum2011/entries/ skepticism/> (édition été 2011).

Lagerlund, Henrik

2010 Rethinking the History of Skepticism: the Missing Medieval Background, Leiden, Brill.

Larivière, D. Anthony et Thomas M. Lennon

2002 «True Believers: the Reception of Descartes's Meditations by Malebranche and Huet», Kriterion, vol. 43, no 106, p. 89-107.

Laursen, John Christian et Richard H. Popkin

1998 «Sources of Knowledge of Sextus Empiricus in Kant's time: A French Translation of Sextus Empiricus from the Prussian Academy, 1779», British Journal for the History of Philosophy, vol. 6, $\mathrm{n}^{\circ} 2$, p. $261-267$.

Lennon, Thomas M.

1999 Reading Bayle, Toronto, University of Toronto Press.

2002a «Did Bayle Read Saint-Evremond?», Journal of the History of Ideas, vol. $63, \mathrm{n}^{\circ} 2$, p. 225-237.

2002b «What Kind of a Skeptic Was Bayle?», Midwest Studies in Philosophy, vol. $26, \mathrm{n}^{\circ} 1$, p. 258-279.

2008 The Plain Truth: Descartes, Huet, and Skepticism, Leiden/Boston, Brill. 
Lennon, Thomas M. et Michael Hickson

2012 «Pierre Bayle», dans Edward N. Zalta, dir., The Stanford Encyclopedia of Philosophy, <http://plato.stanford.edu/archives/fall2012/entries/bayle/> (édition automne 2012).

Marcil-Lacoste, Louise

1982 Claude Buffier and Thomas Reid: Two Common-Sense Philosophers, Kingston, McGill-Queen's University Press.

Menn, Stephen

1998 Descartes and Augustine, Cambridge, Cambridge University Press.

Milton, J. R.

1987 «Induction Before Hume», The British Journal for the Philosophy of Science, vol. 38, no 1, p. 49-74.

Moore, G. E.

1993 Selected Writings, Thomas Baldwin, éd., Londres, Routledge.

Neto, José Raimundo Maia, Gianni Paganini et John Christian Laursen, dir.

2009 Skepticism in the Modern Age: Building on the Work of Richard Popkin, Leiden/Boston, Brill.

Neto, José Raimundo Maia et Richard H. Popkin, dir.

2004 Skepticism in Renaissance and Post-Renaissance Thought: New Interpretations, Amherst (NY), Humanity Books.

Paganini, Gianni et José Raimundo Maia Neto

2009 Renaissance Scepticisms, Dordrecht, Springer.

Perin, Casey

2008 «Descartes and the Legacy of Ancient Skepticism», dans Janet Broughton et John Carriero, dir., A Companion to Descartes, Oxford, Blackwell, p. 52-65.

Perler, Dominik

2004 «Was There a Pyrrhonian Crisis in Early Modern Philosophy? A Critical Notice of Richard H. Popkin», Archiv für Geschichte der Philosophie, vol. $86, \mathrm{n}^{\circ} 2$, p. 209-220.

Popkin, Richard H.

1952 «David Hume and the Pyrrhonian Controversy», The Review of Metaphysics, vol. 6, no 1, p. 65-81.

1963 «Preface», dans H. G. van Leeuwen, The Problem of Certainty in English Thought 1630-1690, La Haye, Martinus Nijhoff, p. 5-9.

1996 «Prophecy and Scepticism in the Sixteenth and Seventeenth century», British Journal for the History of Philosophy, vol. 4, n 1 , p. 1-20.

2003 The History of Scepticism: From Savonarola to Bayle, Oxford, Oxford University Press.

Popkin, Richard H. et Arjo J. Vanderjagt, dir.

1993 Scepticism and Irreligion in the Seventeenth and Eighteenth Centuries, Leiden/New York, Brill. 
Rysiew, Patrick

2005 «Reidian Evidence», Journal of Scottish Philosophy, vol. 3, n 2 , p. 107-121.

Schofield, Malcolm, Myles Burnyeat et Jonathan Barnes, dir.

1980 Doubt and Dogmatism: Studies in Hellenistic Epistemology, Oxford, Clarendon Press/New York (NY), Oxford University Press.

Sedley, David, dir.

2006 Oxford Studies in Ancient Philosophy, Oxford/New York (NY), Oxford University Press.

Silver, Bruce

2002 «Montaigne, An Apology for Raymond Sebond: Happiness and the Poverty of Reason», Midwest Studies in Philosophy, vol. 26, $\mathrm{n}^{\circ} 1$, p. 94-110.

Sinnott-Armstrong, Walter

2004 Pyrrhonian Skepticism, Oxford/New York (NY), Oxford University Press.

Sosa, Ernerst

1980 «The Raft and the Pyramid: Coherence versus Foundations in the Theory of Knowledge», Midwest Studies in Philosophy, vol. 5, nº 1, p. 3-26.

Stroud, Barry

1984 The Significance of Philosophical Scepticism, Oxford, Oxford University Press.

2005 «Comprendre la connaissance humaine en général», dans Pascal Engel et Julien Dutant, dir., Philosophie de la connaissance. Croyance, connaissance, justification, Paris, Vrin, p. 313-344.

Sweet, William

1999 «Paley, Whately, and "Enlightenment Evidentialism"», International Journal for Philosophy of Religion, vol. 45, nº 3, p. 143-166.

Thielemann, L.

1957 «[Review of] Jean-Pierre de Crousaz (1663-1750) et le conflit des idées au siècle des Lumières. By Jacqueline E. de La Harpe [...]», Comparative Literature, vol. 9, $\mathrm{n}^{\circ}$ 1, p. 84-86.

van Leeuwen, Henri G.

1970 The Problem of Certainty in English Thought, 1630-1690, La Haye, Martinus Nijhoff.

Vogt, Katja

2011 «Ancient Skepticism», dans Edward N. Zalta, dir., The Stanford Encyclopedia of Philosophy, $<$ http://plato.stanford.edu/archives/win2011/ entries/skepticism-ancient/> (édition hiver 2011).

Williams, Michael

2010 «Descartes' Transformation of the Sceptical Tradition», dans Richard Bett, dir., The Cambridge Companion to Ancient Scepticism, Cambridge, Cambridge University Press, p. 288-313. 


\section{Dialogue}

\section{Williamson, Timothy}

2000 Knowledge and its Limits, Oxford, Oxford University Press.

Wood, Allen

2007 «The Duty to Believe According to the Evidence», International Journal for Philosophy of Religion, vol. 63, $\mathrm{n}^{\text {os }} 1-3, \mathrm{p} .7-24$. 